\title{
OPEN Machine learning approach to integrated endometrial transcriptomic datasets reveals biomarkers predicting uterine receptivity in cattle at seven days after estrous
}

\author{
Maria B. Rabaglino \& Haja N. Kadarmideen ${ }^{\bowtie}$
}

The main goal was to apply machine learning $(\mathrm{ML})$ methods on integrated multi-transcriptomic data, to identify endometrial genes capable of predicting uterine receptivity according to their expression patterns in the cow. Public data from five studies were re-analyzed. In all of them, endometrial samples were obtained at day 6-7 of the estrous cycle, from cows or heifers of four different European breeds, classified as pregnant $(n=26)$ or not $(n=26)$. First, gene selection was performed through supervised and unsupervised ML algorithms. Then, the predictive ability of potential key genes was evaluated through support vector machine as classifier, using the expression levels of the samples from all the breeds but one, to train the model, and the samples from that one breed, to test it. Finally, the biological meaning of the key genes was explored. Fifty genes were identified, and they could predict uterine receptivity with an overall $96.1 \%$ accuracy, despite the animal's breed and category. Genes with higher expression in the pregnant cows were related to circadian rhythm, Wnt receptor signaling pathway, and embryonic development. This novel and robust combination of computational tools allowed the identification of a group of biologically relevant endometrial genes that could support pregnancy in the cattle.

Various bioinformatics and systems biology tools in animal production and health sciences ${ }^{1}$, and specifically in cattle artificial reproduction ${ }^{2}$, focus on integrating biological data layers (genomics and transcriptomics) and application of statistical-bioinformatics methods (e.g. eQTL mapping) to identify functionally relevant targets and biomarkers. However, the emergence of machine learning (ML), as a big data science tool, is less explored in livestock functional genomics in general, and bovine species in particular. ML refers to the use of self-learning algorithms to make sense of big data and is a branch of artificial intelligence that holds great potential for pattern recognition in complex datasets ${ }^{3,4}$, such as the ones derived from the "omics" technologies. In transcriptomic data (captured by either microarrays or RNA-sequencing platforms), expression pattern analysis is central to find functionally relevant groups of genes under different treatment conditions or phenotypic categories. Thus, application of ML tools represents a powerful analytical approach that can be strengthened when it is applied to data integrated from several datasets (i.e., multi-transcriptomic data), which provides a robust overview of the system under study ${ }^{5}$.

Here, we investigated ML methods across multi-transcriptomic data, in the context of characterizing the receptive endometrium at the time of embryo transfer (ET) in European cattle. The endometrial transcriptomic profile should determine a favorable environment for contact and communication with the embryo at around 5-6 days of pregnancy, when the conceptus reaches the uterus ${ }^{6}$. Thus, identification of a receptive endometrium becomes crucial at around 7 days of the estrous cycle, when an embryo is deposited into the uterus after the application of assisted reproductive technologies. Previous works, including ours ${ }^{7}$, have demonstrated that the

Quantitative Genetics, Bioinformatics and Computational Biology Group, Department of Applied Mathematics and Computer Science, Technical University of Denmark, Richard Petersens Plads, Building 324, 2800 Kgs. Lyngby, Denmark. ${ }^{\bowtie}$ email: hajak@dtu.dk 


\begin{tabular}{|c|c|c|c|c|c|c|c|}
\hline $\begin{array}{l}\text { Accession number } \\
\text { in GEO }\end{array}$ & Animal breed & Animal category & $\begin{array}{l}\text { Method to induce } \\
\text { pregnancy }\end{array}$ & $\begin{array}{l}\text { Method of sample } \\
\text { collection }\end{array}$ & Number of samples & $\begin{array}{l}\text { Platform for } \\
\text { transcriptomic } \\
\text { determination }\end{array}$ & $\begin{array}{l}\text { Authors \& date of } \\
\text { data publication }\end{array}$ \\
\hline GSE115756 & Holstein & $\begin{array}{l}\text { Lactating cows (1st to } \\
\text { 3rd lactation) }\end{array}$ & IVP-ET & Biopsy instrument & $\begin{array}{l}\mathrm{R}=8 \\
\text { non } \mathrm{R}=9\end{array}$ & $\begin{array}{l}\text { Illumina HiSeq } 2500 \\
\text { (RNA- sequencing) }\end{array}$ & $\begin{array}{l}\text { Mazzoni et al. }{ }^{7}-\text { Dec } \\
31,2019\end{array}$ \\
\hline GSE107741 & Japanese Black & Cows & AI / IV-ET & Biopsy instrument & $\begin{array}{l}\mathrm{R}=6 \\
\text { non } \mathrm{R}=5\end{array}$ & $\begin{array}{l}\text { Agilent-023647 B. tau- } \\
\text { rus Oligo Microarray v2 }\end{array}$ & $\begin{array}{l}\text { Matsuyama et al. (article } \\
\text { not published)_Jan } \\
11,2018\end{array}$ \\
\hline GSE29853 & Charolais $\times$ Limousine & Heifers & AI & $\begin{array}{l}\text { Postmortem peeling } \\
\text { from the uterine myo- } \\
\text { metrium }\end{array}$ & $\begin{array}{l}\mathrm{R}=6 \\
\text { non } \mathrm{R}=6\end{array}$ & \multirow{3}{*}{$\begin{array}{l}\text { Affymetrix Bovine } \\
\text { Genome Array }\end{array}$} & $\begin{array}{l}\text { Killen et al. }{ }^{11}-\text { Jun } \\
10,2011\end{array}$ \\
\hline GSE36080 & \multirow[t]{2}{*}{ Simmental } & \multirow[t]{2}{*}{ Heifers } & \multirow[t]{2}{*}{ IV-ET } & \multirow[t]{2}{*}{ Cytobrush } & $\begin{array}{l}\mathrm{R}=3 \\
\text { non } \mathrm{R}=3\end{array}$ & & $\begin{array}{l}\text { Ponsuksili and } \\
\text { Wimmers' }- \text { Jan 01, } \\
2013\end{array}$ \\
\hline GSE20974 & & & & & $\begin{array}{l}\mathrm{R}=3 \\
\text { non } \mathrm{R}=3\end{array}$ & & $\begin{array}{l}\text { Salilew-Wondim } \\
\text { et al. }{ }^{55} \text {-Mar 25, } 2010\end{array}$ \\
\hline
\end{tabular}

Table 1. Characteristics of each dataset selected for data integration and analysis. For all the experiments, endometrial samples from Bos taurus cattle were obtained around day 7 of the estrous cycle, and they were classified retrospectively or prospectively according to the pregnancy results. IVP-ET: transfer of in-vitro produced embryos; IV-ET: transfer in-vivo produced embryos; AI: artificial insemination; R and nonR: animals classified as receptive or not, respectively.

endometrial transcriptomic profile at day 6 or 7 differs between animals that become pregnant or not. This fact has been shown in Nellore cows after artificial insemination $(\mathrm{AI})^{8}$, Simmental heifers after in vivo produced $\mathrm{ET}^{9,10}$, dairy cows after in-vitro produced $\mathrm{ET}^{7}$, and cross-breed heifers after $\mathrm{AI}^{11}$. These studies have applied high-throughput technologies with the goal of determining the differentially expressed genes in the endometria of animals that resulted pregnant compared to those that do not. Their results helped to shed light on the identification of those genes whose expressions control the fate of uterine receptivity. Nevertheless, results from these studies are not entirely consistent, since several other factors, such as breed and category, can influence gene expression. Therefore, the identification of endometrial genes as biomarkers of receptivity is still challenging.

In the present study, we combined data integration with supervised and unsupervised ML tools to provide actionable knowledge from various endometrial transcriptomic datasets. We hypothesized that such novel computational approach could reveal the main gene expression signature of the receptive endometrium at the time of ET in cattle. The goal of this study was to conduct multi-transcriptomic data integration from five publicly available datasets, including our recent study on endometrial transcriptomics ${ }^{7}$, and apply ML tools to such integrated data, to identify biomarker genes determining uterine receptivity according to their expression patterns. These studies, listed in Table 1, have in common that endometrial samples from European or Taurine cattle (Bos taurus taurus) were obtained at day 6-7 of the estrous cycle, and they were classified as receptive $(\mathrm{R}, \mathrm{n}=26)$ or not (nonR, $n=26)$ after ET or AI, depending on the study. Selection and validation of the potential biomarkers were done in three steps involving supervised and unsupervised ML methods. Once these key genes were determined, the final aim was to explore their biological characteristics through predictions in external data to discern the role of estradiol and progesterone in their expression, and network analysis to reveal related genes.

\section{Results}

In what follows below, the main results are categorized into four topics.

Identification of groups of potential biomarker genes through supervised ML. The software BioDiscML ${ }^{12}$ was employed for selection of potential biomarker genes. This software automates the main steps in ML by implementing methods for features and model selection, in order to identify the best model for data classification. The software generated 2097 models, from which only five models presented accuracy higher than $90 \%$ in the test set and in the evaluation procedures in the train set. These models were:

a. Two models of Bayes Network optimized by accuracy of prediction, which selected 100 and 75 genes

b. Two multinomial logistic regression models, also optimized by accuracy of prediction, which selected the same 100 and 75 genes, and

c. Bayes Network optimized by False Discovery Rate, which selected 50 genes.

The 100, 75 and 50 selected genes were overlapping, meaning that the 50 genes were repeated in the three groups.

Identification of the best group of biomarker genes through unsupervised ML. The groups of genes identified as potential biomarkers were evaluated for their ability to blindly cluster apart the $\mathrm{R}$ and nonR samples, according to their expression levels, in a hierarchical clustering. As expected, the group of 50 genes showed the best performance (92.3\% accuracy). Only one nonR sample from the Holstein cows (nonR_Hols_6) was clustered with the R samples, while three $\mathrm{R}$ samples were grouped with the nonR samples. These samples were one from Holstein (R_Hols_3), one from the Charolais x Limousine (R_Cont_3) and one from the second 
study with the Simmental heifers (R_Sim2_1). The corresponding dendrograms and heat maps for the expression signatures are shown in Supplementary Fig. 1, together with the confusion matrix and accuracy for each classification.

In addition, hierarchical clustering of the genes according to their expression showed two main clusters of genes, corresponding to those with increased or decreased expression in the R cows (up-regulated or downregulated, respectively).

Table 2 lists the 50 genes, with the respective indication if they were more (UP, $n=32)$ or less (DOWN, $n=18$ ) expressed in the 7-day endometria of the $\mathrm{R}$ animals.

Validation of the selected set of biomarker genes through supervised ML. The next step was to verify if the expression signature of the 50 selected genes were able to predict uterine receptivity. For this, we applied Support vector machines (SVM) as classifier, using all the samples but the samples from a given breed as training set, and the samples from such breed as testing set. Therefore, we could discern if the expression signature of these genes would be able to predict uterine receptivity across all the bovine breeds.

The evaluation metrics associated with the confusion matrix for each of the four train/test set are depicted in Table 3. Using the expression of the 50 genes in all the samples, but the samples for a particular breed, to train the SVM, the accuracy to predict correctly the uterine receptivity in that particular breed was $100 \%$ for the Japanese and Simmental breeds, $94.1 \%$ for the Holstein cows and 91.7\% for Charolais x Limousine heifers. One nonR sample from the Holstein cows (non_R_6) was misclassified as R, while one R sample from the Charolais $\mathrm{x}$ Limousine heifers was misclassified as nonR ( $\mathrm{R} \_$Cont_1). Therefore, the overall accuracy was $96.1 \%$.

Determination of the biological significance of the selected biomarker genes. As a final step, we investigated the biological meaning of the 50 genes through two methods: predictions in external datasets and functional/network analysis.

Predictions in external datasets: with the aim of understanding the role of estradiol and progesterone in the expression of the biomarker genes, two datasets were selected to generate the test sets based on the endometrial expression of the 50 biomarker genes. The training set consisted on the expression of the 50 genes in the 52 samples described in Table 1. Predictions of 'receptive' samples for each test set were as follow:

Test set 1) Five out of five pregnant heifers with normal progesterone levels (PN), but only one out of five pregnant heifers treated with a progesterone device on day 3 of the estrous cycle (PH).

Test set 2) Three out of three ovariectomized cows receiving a progesterone treatment for six days plus estradiol at day $6(\mathrm{E} 2+\mathrm{P} 4)$ but none out of three receiving only the progesterone treatment $(\mathrm{P} 4)$.

Accordingly, samples that were classified as 'receptive' (PN and E2 + P4) tended to cluster with the R samples, and vice versa for samples classified as 'non receptive' (PH and P4), in a PCA plot (Fig. 1).

Functional/network analysis: functional classification of the protein class for each gene was performed with the Panther database ${ }^{13}$ and network analysis was done with the Cytoscape software (V. 3.7.2) ${ }^{14}$.

From the 50 genes, 26 genes were classifying into protein classes, from which the most abundant protein class was gene-specific transcriptional regulator (six out of the 26). These regulators were the following transcription factors: Cellular tumor antigen p53 (TP53), Basic helix-loop-helix family member e40 (BHLHE40), Hematopoietically expressed homeobox (HHEX) and Zinc finger and SCAN domain containing 12 (ZSCAN12). The following regulators are transcription co-factors: Transducin like enhancer of split (TLE4) and C-terminalbinding protein 2 (CTBP2). All the genes were more expressed in the animals that become pregnant, except for ZSCAN12 and CTBP2.

The top 100 related genes to the up- and down-regulated biomarker genes in the $\mathrm{R}$ animals were inferred and analyzed with Cytoscape (Supplementary Table 1). These genes generated highly connected networks (Supplementary Fig. 2). The significantly enriched biological processes (adj. $p<0.05$ ) related to these networks are listed in Supplementary Table 2. The main significant biological processes determined in the network derived from the up-regulated genes in the endometria of the $\mathrm{R}$ cows are: positive regulation of biological/cellular process, regulation of gene expression, circadian rhythm, regulation of apoptosis, Wnt receptor signaling pathway, and embryonic development. For the down-regulated genes, the main non-redundant biological processes are: chromosome segregation, lipid modification, negative regulation of biological/cellular process, M phase of cell cycle, and fatty acid oxidation.

\section{Discussion}

So far, most of the studies of the bovine endometrial transcriptome during the early-luteal phase period have utilized bioinformatics methods to detect differentially expressed genes between the groups in that particular study $^{7-10,15}$. The outputs of these investigations are deposited in the public GEO database, which enables the access to a large amount of high-throughput data ${ }^{16}$. Integration of several datasets could lead to a better characterization of the system under study ${ }^{5}$, as done, for example, for the human endometrial transcriptome ${ }^{17}$. On the other hand, ML algorithms have emerged as useful tools to recognize patterns in data generated by "omics" assays ${ }^{4}$. Here, we demonstrated the power of combining data integration and ML methods to detect endometrial genes whose expression patterns potentially identify a receptive endometrium at around seven days of the estrous cycle.

In the cow, the pre-implantation period is so critical that more than $70 \%$ of pregnancy failure associated with embryo death occurs here ${ }^{18}$. This represents one of the main causes of economic loss and thus, the understanding of the early physiological changes occurring in the endometrium, which are determined by variations in the endometrial transcriptome, takes major importance. In the present study, we integrated endometrial transcriptomic data from the Bos taurus taurus during this early-luteal phase, with the main aim of identifying a group or set of genes characterizing a receptive endometrium despite the breed and category. Data from Bos taurus indicus were 


\begin{tabular}{|c|c|c|c|}
\hline Ensembl gene ID & Gene symbol & Gene name & Direction \\
\hline ENSBTAG00000001069 & TP53 & Tumor protein $\mathrm{p} 53$ & UP \\
\hline ENSBTAG00000001568 & PPIC & Peptidylprolyl isomerase C & UP \\
\hline ENSBTAG00000002108 & YWHAQ & $\begin{array}{l}\text { Tyrosine 3-monooxygenase/tryptophan 5-monooxygenase activation protein } \\
\text { theta }\end{array}$ & UP \\
\hline ENSBTAG00000002130 & SMPD4 & Sphingomyelin phosphodiesterase 4 & UP \\
\hline ENSBTAG00000003397 & CTBP2 & C-terminal binding protein 2 & DOWN \\
\hline ENSBTAG00000003532 & TLE4 & Transducin like enhancer of split 4 & UP \\
\hline ENSBTAG00000003718 & HACL1 & 2-hydroxyacyl-CoA lyase 1 & DOWN \\
\hline ENSBTAG00000003843 & SMARCAL1 & $\begin{array}{l}\text { SWI/SNF related, matrix associated, actin dependent regulator of chromatin, } \\
\text { subfamily a like } 1\end{array}$ & UP \\
\hline ENSBTAG00000004459 & TMEM45A & Transmembrane protein $45 \mathrm{~A}$ & UP \\
\hline ENSBTAG00000004769 & NEIL2 & Nei like DNA glycosylase 2 & DOWN \\
\hline ENSBTAG00000005092 & ROR2 & Receptor tyrosine kinase like orphan receptor 2 & UP \\
\hline ENSBTAG00000005462 & FXR2 & FMR1 autosomal homolog 2 & DOWN \\
\hline ENSBTAG00000006002 & DIDO1 & Death inducer-obliterator 1 & UP \\
\hline ENSBTAG00000007007 & WDR20 & WD repeat domain 20 & DOWN \\
\hline ENSBTAG00000008083 & SEL1L & SEL1L ERAD E3 ligase adaptor subunit & UP \\
\hline ENSBTAG00000008181 & CHAF1A & Chromatin assembly factor 1 subunit $\mathrm{A}$ & UP \\
\hline ENSBTAG00000008943 & ZSCAN12 & Zinc finger and SCAN domain containing 12 & DOWN \\
\hline ENSBTAG00000009121 & STAG2 & Stromal antigen 2 & DOWN \\
\hline ENSBTAG00000009541 & SUCLG2 & Succinate-CoA ligase GDP-forming beta subunit & DOWN \\
\hline ENSBTAG00000009863 & BHLHE40 & Basic helix-loop-helix family member e40 & UP \\
\hline ENSBTAG00000010416 & RIN3 & Ras and Rab interactor 3 & UP \\
\hline ENSBTAG00000011205 & PPP1R42 & Protein phosphatase 1 regulatory subunit 42 & UP \\
\hline ENSBTAG00000011818 & COL26A1 & Collagen type XXVI alpha 1 chain & UP \\
\hline ENSBTAG00000012454 & SLC35A3 & Solute carrier family 35 member A3 & DOWN \\
\hline ENSBTAG00000014217 & HHEX & Hematopoietically expressed homeobox & UP \\
\hline ENSBTAG00000014393 & CLK2 & CDC like kinase 2 & UP \\
\hline ENSBTAG00000014644 & DUS2 & Dihydrouridine synthase 2 & UP \\
\hline ENSBTAG00000014713 & RARRES1 & Retinoic acid receptor responder 1 & UP \\
\hline ENSBTAG00000014838 & VPS26B & VPS26, retromer complex component B & DOWN \\
\hline ENSBTAG00000015390 & FN3KRP & Fructosamine 3 kinase related protein & DOWN \\
\hline ENSBTAG00000016977 & FUNDC2 & FUN14 domain containing 2 & UP \\
\hline ENSBTAG00000017505 & PAXIP1 & PAX interacting protein 1 & UP \\
\hline ENSBTAG00000017833 & RNF19A & Ring finger protein 19A, RBR E3 ubiquitin protein ligase & DOWN \\
\hline ENSBTAG00000019155 & FRS2 & Fibroblast growth factor receptor substrate 2 & DOWN \\
\hline ENSBTAG00000020611 & GLB1L & Galactosidase beta 1 like & UP \\
\hline ENSBTAG00000020943 & CTU2 & Cytosolic thiouridylase subunit 2 & DOWN \\
\hline ENSBTAG00000021151 & MYH10 & Myosin heavy chain 10 & UP \\
\hline ENSBTAG00000021680 & SKA2 & Spindle and kinetochore associated complex subunit 2 & UP \\
\hline ENSBTAG00000021768 & CCNG2 & Cyclin G2 & DOWN \\
\hline ENSBTAG00000023179 & TRIB1 & Tribbles pseudokinase 1 & UP \\
\hline ENSBTAG00000024240 & ACADM & acyl-CoA dehydrogenase, C-4 to C-12 straight chain & DOWN \\
\hline ENSBTAG00000026290 & PIK3C3 & Phosphatidylinositol 3-kinase catalytic subunit type 3 & DOWN \\
\hline ENSBTAG00000031385 & RFWD2 & Ring finger and WD repeat domain 2 & UP \\
\hline ENSBTAG00000032613 & SCG5 & Secretogranin $\mathrm{V}$ & UP \\
\hline ENSBTAG00000034693 & SYT1 & Synaptotagmin 1 & UP \\
\hline ENSBTAG00000037757 & EBF4 & Early B-cell factor 4 & UP \\
\hline ENSBTAG00000038866 & UBE2I & Ubiquitin conjugating enzyme E2 I & UP \\
\hline ENSBTAG00000039980 & CLECL1 & C-type lectin-like 1 & UP \\
\hline ENSBTAG00000043964 & ARL5B & ADP ribosylation factor like GTPase 5B & DOWN \\
\hline ENSBTAG00000045550 & TSPAN6 & Tetraspanin 6 & UP \\
\hline
\end{tabular}

Table 2. List of the 50 endometrial genes identified as biomarkers to determine pregnancy status around day 7 of the estrous cycle in the Bos taurus cattle. The column named "direction" indicates if the gene was more (UP) or less (DOWN) expressed in the endometria of the animals that resulted pregnant. 


\begin{tabular}{|l|l|l|l|l|}
\hline Metric & Holstein & Charolais $\times$ Limousine & Japanese black & Simmental \\
\hline Accuracy & 0.941 & 0.917 & 1.000 & 1.000 \\
\hline Kappa & 0.883 & 0.833 & 1.000 & 1.000 \\
\hline Accuracy Lower & 0.713 & 0.615 & 0.715 & 0.735 \\
\hline Accuracy Upper & 0.999 & 0.998 & 1.000 & 1.000 \\
\hline Accuracy Null & 0.529 & 0.500 & 0.545 & 0.500 \\
\hline Accuracy PValue & 0.000 & 0.003 & 0.001 & 0.000 \\
\hline McNemar PValue & 1.000 & 1.000 & NA & NA \\
\hline Sensitivity & 0.889 & 1.000 & 1.000 & 1.000 \\
\hline Specificity & 1.000 & 0.833 & 1.000 & 1.000 \\
\hline Positive Predictive Value & 1.000 & 0.857 & 1.000 & 1.000 \\
\hline Negative Predictive Value & 0.889 & 1.000 & 1.000 & 1.000 \\
\hline Precision & 1.000 & 0.857 & 1.000 & 1.000 \\
\hline Recall & 0.889 & 1.000 & 1.000 & 1.000 \\
\hline F1 & 0.941 & 0.923 & 1.000 & 1.000 \\
\hline Prevalence & 0.529 & 0.500 & 0.455 & 0.500 \\
\hline Detection Rate & 0.471 & 0.500 & 0.455 & 0.500 \\
\hline Detection Prevalence & 0.471 & 0.583 & 0.455 & 0.500 \\
\hline Balanced Accuracy & 0.944 & 0.917 & 1.000 & 1.000 \\
\hline
\end{tabular}

Table 3. Evaluation metrics corresponding to the classifications on pregnancy status based on the expression of the 50 endometrial genes for each breed, using Support Vector Machine as classifier, trained with all the samples except for the samples of the particular breed.

(A)

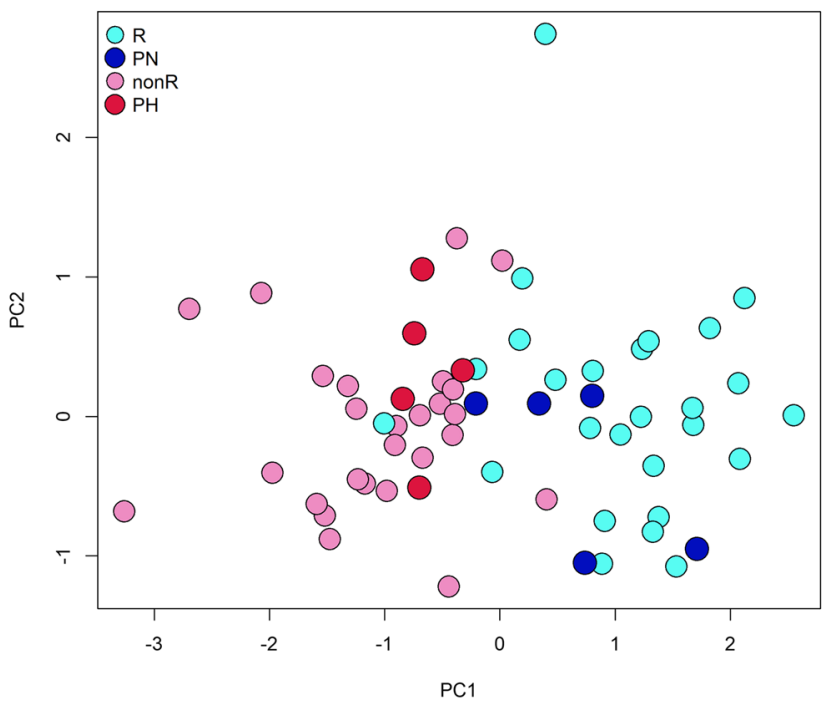

(B)

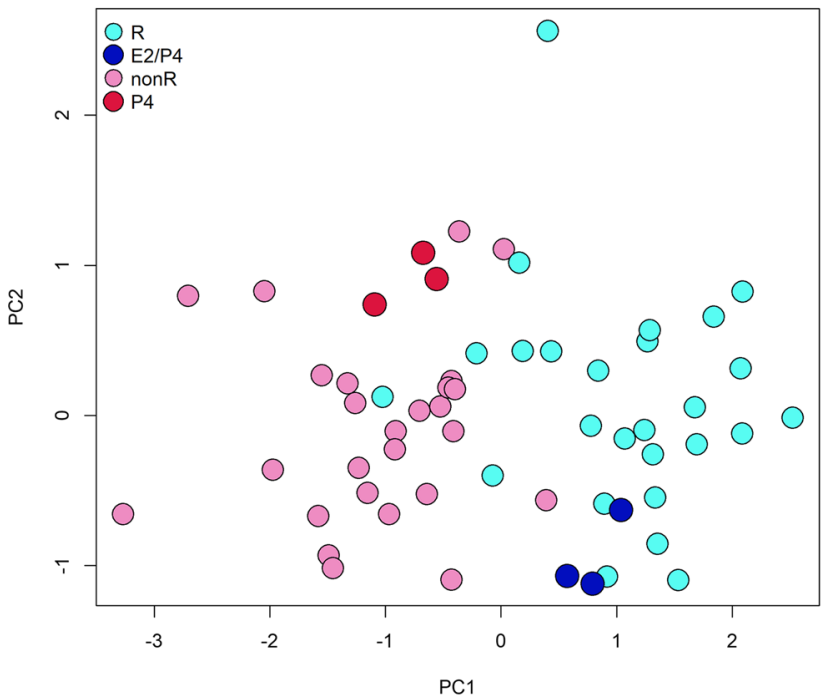

Figure 1. Principal component analysis of samples' distribution according to the expression of the 50 biomarker genes. The plots show the distribution of samples collected from receptive (R) and non-receptive (nonR) cows, and samples obtained from: (A) pregnant cows but treated with a progesterone device from day 3 $(\mathrm{PH})$ or with normal progesterone levels $(\mathrm{PN}) ;(\mathrm{B})$ ovariectomized cows receiving a progesterone treatment for 6 days plus estradiol at day $6(\mathrm{E} 2+\mathrm{P} 4)$ or only the progesterone treatment $(\mathrm{P} 4)$.

not considered to avoid confounding differences given the bifurcation in the phylogenetic tree. Selected datasets (Table 1) shared similar experimental designs. Except for the dataset GSE29853, the other datasets classified the animals retrospectively according to pregnancy status after the biopsy (although GSE107741 was based on the results before and after the biopsy). For the dataset GSE29853, the authors classified the animals based on the results of previous AI. Therefore, all these public datasets have in common that consist of endometrial samples obtained at around day 7 of the estrous cycle, samples were classified according to pregnancy status, and the transcriptome was measured through a high-throughput technology. The application of bioinformatics procedures allowed the integration of these datasets, in the sense that technical differences given by the platform employed 
for transcriptomic measurement, or the experiment itself, were eliminated through a data pre-processing step (Supplemental Fig. 3).

As per our main objectives, we applied a series of ML procedures (that included identification of sets of genes and application of unsupervised and supervised ML tools to determine the best one) to determine a group of 50 genes with the capability to predict pregnancy status according to their expression levels (Table 2). Even more, the expressions of these genes in all the samples but a particular breed were able to predict uterine receptivity with an overall $96.1 \%$ accuracy, validating the predictive capability of these key genes (Table 3 ).

Between them, there were six transcriptional regulators, corresponding to four transcription factors and two co-factors. Furthermore, five of these 50 genes have been associated with cow fertility in recent studies using genome-wide association analysis. YWHAQ and PAXIP1 were identified as master regulators (molecules that have indirect relationships to positional candidate genes through upstream regulators), and SUCLG2 as one positional candidate gene, associated with dairy heifer fertility ${ }^{19}$. TP53, one of the transcription factors discussed below, was one of the three top upstream regulators of positional candidate genes, while MYH10 a regulator target gene, associated with beef cattle fertility ${ }^{20}$.

It is well accepted that progesterone concentrations regulate the endometrial expression of genes determining uterine receptivity, and it plays a key role in pregnancy establishment and conceptus development ${ }^{21,22}$. Therefore, to explore the action of this hormone on the expression of these 50 genes, additional external data from two studies were re-analyzed. In one study, pregnant heifers presented normal or high levels of progesterone from day 3 of the estrous cycle ${ }^{23}$. Although all heifers were pregnant, only one from the high-progesterone group was classified as such according to the expression of the 50 genes (Fig. 1A). Forde et al. ${ }^{23}$ concluded that progesterone supplementation during early pregnancy advances endometrial gene expression in cattle, and so the endometria of those animals probably reflected changes occurring later in the estrous cycle. In the other study, ovariectomized cows were treated with progesterone for six days, receiving an injection of estradiol benzoate at the end of the treatment or not $^{24}$. Only samples obtained from animals receiving the estradiol treatment were classified as receptive, according to the expression of the 50 genes (Fig. 1B).

Thus, these results suggest that the expression of the 50 genes is temporally regulated and their differences in expression between $\mathrm{R}$ and nonR animals would occur at around day 7 of the estrous cycle but not later in the cycle. Also, these genes probably are responding to the increasing estradiol levels of the first wave together with the increased levels of progesterone ${ }^{25}$, but not to progesterone alone, although this fact should be confirmed by experimental studies in vivo.

In addition, and to explore more deeply the biological significance of the 50 genes, related genes were inferred in a network analysis (Supplementary Fig. 2). These related genes might not behave as crucial genes during the early luteal phase period, but their expression, or their products, or the pathway(s) they shared with the key genes, could be affected later. We cannot know exactly the number of genes that would be regulated by the key genes, and so the arbitrary top 100 genes were explored, together with the significantly enriched biological processes by these genes, respectively for the biomarker genes that showed higher or lower expression in the R cows.

One of the biomarker genes with higher expression in the R cows was TP53, which is well known as tumor suppressor because its protein (p53) regulates cell division by keeping cells from growing and dividing (proliferating) too fast or in an uncontrolled way. In response to different stress signals, p53 can hold cell division in both the G1/S phase and G2/M phase checkpoints, in order to prevent chromosomal replication specifically during the cell cycle if DNA damage is present, and even to induce cell apoptosis ${ }^{26,27}$. The actions of p53 are critical to avoid tumor development, but it also regulates many cellular processes, including metabolism, antioxidant response, and DNA repair ${ }^{26}$. Interestingly, regulation of transcription and cell death/apoptosis were biological processes enriched by the related genes to the ones with higher expression in the R cows, while $M$ phase of the cell cycle, chromosome segregation and lipid oxidation to the ones with lower expression in the R cows. Furthermore, many steps involved in implantation in the human, such as apoptosis and angiogenesis, are regulated by $\mathrm{p} 53$ and thus this protein could play a broader role in the survival of the specie by optimizing the embryo implantation ${ }^{28}$. This study suggests that early expression by TP53 is critical for uterine receptivity in the cow as well. In the report from Ponsuksili et al. ${ }^{9}$, the authors found that activated TP53 was associated with the endometria of high receptive cows, although on day 3 (not on day 7). Thus, the role of TP53 expression during the early-luteal phase in the bovine endometrium deserves further investigation.

Another biological process enriched with the related genes to the ones with higher expression in the R cows, was Wnt signaling pathway. The key genes involved in it were TLE4 and ROR2. On the other hand, CTBP2, a transcription co-factor with higher expression in the nonR cows, also participates in the Wnt pathway. TLE4 is a transcriptional co-repressor whose products, and the ones encoded by the TLE1-3, inhibit the transcriptional activation mediated by the nuclear $\beta$-catenin CTNNB1 and TCF family members in the canonical Wnt signaling pathway. Conversely, HHEX, a transcription factor more expressed in the R cows, acts early in embryo development to enhance canonical WNT-signaling by repressing expression of TLE4 ${ }^{29}$. ROR2 signals through a Wnt responsive, $\beta$-catenin independent pathway and suppress a canonical $W n t / \beta$-catenin signal ${ }^{30}$. Finally, CTBP2 associates with major components of the $\beta$-catenin destruction complex and limits the accessibility of $\beta$-catenin to core transcription factors in undifferentiated embryonic stem cells, which allows exit from pluripotency ${ }^{31}$. In the cow, maternally derived Wnt are important for the development of the preimplantation embryo ${ }^{32}$. Therefore, the expression of these biomarker genes identified in the present study could play a crucial role in the regulation of the canonical and non-canonical Wnt pathway in the early-diestrous endometrium.

Lastly, one more biological process that deserves attention is the circadian rhythm, influenced by the transcription factor BHLHE40. The basic helix-loop-helix protein encoded by this gene interacts with the clock genes and modulates the circadian phase of the clock genes, playing a role in the fine regulation and robustness of the molecular clock ${ }^{33,34}$. This clock is highly important in reproductive tissues, including the regulation of the 
uterine function, although more studies are needed to define its role in the endometrial receptivity (reviewed by Sen and Hoffmann ${ }^{35}$ ).

Study strengths and limitations: This work embraces the output from five studies employing four breeds with distinct purposes (dairy, beef and double) and different techniques for sample collection, which are factors that can influence endometrial gene expression. However, the relative difference of expression between $\mathrm{R}$ and nonR animals, for all the biomarker genes (except for five) was similar in all the breeds before correction for the experimental effect (Supplementary Fig. 4). In other words, if samples are taken from a given breed cattle, using the same technique, and at around day 7 of the estrous cycle, these genes are expected to show differences in expression between $\mathrm{R}$ and nonR animals. Even when the differences were subtle, the overall behavior of these key genes would help to define those animals with a higher uterine capacity to support pregnancy.

On the other hand, the establishment of pregnancy is a complex process that depends not only on the receptive endometrium but also on embryonic viability and synchrony of actions between both parts (reviewed by Spenser et al. ${ }^{21}$ ). Therefore, we cannot expect that the sole expression of these 50 genes, identified by mathematical approaches, could determine animals that would become pregnant or not which such high accuracy. However, we believe that our results could be of enormous help to understand the characteristics of a receptive endometrium at the time of ET and provide the basis for further studies.

\section{Conclusion}

In summary, the application of supervised and unsupervised ML approaches for multi-transcriptomic data integration and target/gene selection, allowed the identification of a group of 50 endometrial genes with high predictive capability (96.1\%) to define uterine receptivity in Taurine cattle at around seven days of the estrous cycle, despite the animal's breed and category. From a data science perspective, results show the scope and power of ML methods in multi-transcriptomic studies and from a biological perspective, results highlight the concept of the strong influence of the maternal environment for pregnancy establishment, which is determined independently of the presence of the embryo.

\section{Methodology}

High-throughput datasets. Five transcriptomic datasets were downloaded from a public functional genomic data repository: Gene Expression Omnibus (GEO) from the National Center for Biotechnology Information $^{36,37}$. These studies were selected because they all have in common that endometrial samples from Bos taurus taurus animals were obtained at day 6-7 of the estrous cycle, and they were classified as pregnant $(n=26)$ or not $(n=26)$ after ET or AI, depending on the study. The accession number and main characteristics of each dataset are shown in Table 1.

Only our dataset (GSE1 15756, ${ }^{7}$ ) used the RNA-sequencing technology (Illumina HiSeq 2500 platform). The other datasets measured gene expression through the microarray technology. The study GSE107741 used the Agilent-023647 B. taurus (Bovine) Oligo Microarray v2 while the other three (GSE29853, GSE36080, GSE20974) employed the Affymetrix Bovine Genome Array platform.

Data integration. The $\mathrm{R}$ software platform ${ }^{38}$ was employed in the following procedures. The raw counts obtained from the RNA-sequencing in our data were transformed through the variance stabilizing transformation method ${ }^{39}$, using the vst function from the DESeq2 package for $\mathrm{R}^{40}$. This transformation removes the dependence of the variance on the mean and produces transformed data on the log 2 scale, which has been normalized with respect to library size or other normalization factors. The raw data obtained from samples hybridized to the Affymetrix or Agilent platforms were processed with the gcRMA ${ }^{41}$ or limma ${ }^{42}$ packages, respectively. Data were imported into R, background corrected, and then transformed and normalized using the quantile normalization method. Next, rows of each data set were collapsed, in order to retain the microarray probe with the highest mean value from the group of the genes with the same Ensembl ID.

Therefore, a table with transformed and normalized gene expression values for each sample was generated for each of the five studies, using the same identifier for the transcripts (Ensembl ID). These tables were integrated into a single table containing the expression of 9850 annotated transcripts for the 52 samples in total (only transcripts with expression values for all the samples were retained). Next, the batch effects (i.e., the fact that the data were obtained from different studies) were removed with the ComBat function from the sva package ${ }^{43}$. A multidimensional scaling analysis (MDS) was employed to evaluate between samples similarities before and after the batch removal (Supplemental Fig. 3), with the Glimma package ${ }^{44}$.

Selection of biomarker genes through supervised ML. The details about each step followed by the BioDiscML software ${ }^{12}$ are specified in the reference and in the GitHub page (https://github.com/mickaelleclercq /BioDiscML). Briefly, a first sampling step separates the data into a train and a test set $(2 / 3$ and $1 / 3$, respectively, by default), that are later used to assess the model, or the user can define these datasets. We chose this last option instead of using a random separation of the data in order to have samples from all the breeds on each set. The training set consisted of 34 samples (11 from Holstein, 7 from Japanese Black, and 8 from Charolais x Limousine, or Simmental cows, respectively). The test set consisted of 18 samples (6 from Holstein, 4 from Japanese Black, and 4 from Charolais x Limousine, or Simmental cows, respectively).

As second step, a feature-ranking algorithm sorts the features (or genes) based on their predictive power with respect to the class ( $\mathrm{R}$ or nonR), retaining only the best 1000 genes. Next, two methods are employed for searching and selecting the potential biomarker genes: top $k$ features and stepwise, for each ML algorithm and each optimization evaluation criterion. At each iteration, the created model is evaluated by tenfold cross validation and the selected genes are retained if the predictive performance is improved. When the signatures of 
biomarker genes are identified, the models are evaluated again. Finally, it is possible to let the software to select the best model (or combine the best ones), or this step can be done manually. For this, one of the output files describes each model with its associated performance metrics and the list of corresponding genes.

For this study, we manually selected those models that resulted with a prediction accuracy higher than $90 \%$ in the test set and the following evaluations' procedures in the train set: tenfold cross validation, leave-one-out cross validation, repeated holdout and bootstrapping; and repeated holdout in the whole set.

Identification of the best group of biomarker genes through unsupervised ML. The groups of genes identified as potential biomarkers were evaluated for their ability to blindly cluster apart the $\mathrm{R}$ and nonR samples according to their expression levels. For this, a hierarchical clustering was employed, using Spearman Rank Correlation as similarity metric and complete linkage as clustering method, implemented with the Cluster 3.0 software ${ }^{45}$. The resulting dendrogram and the heat map were visualized with Java TreeView ${ }^{46}$.

The correct clustering of the $\mathrm{R}$ and nonR samples for each group of genes was evaluated using a confusion matrix, selecting the genes that, according to their expression, presented the highest accuracy to cluster apart the samples from each group.

Validation of the selected set of biomarker genes through supervised ML. Once the set of potential biomarker genes was selected according to unsupervised learning, the next step was to verify if the expression levels of these genes were able to predict pregnancy status. For this, we applied a different ML model than the ones identified by the BioDiscML software, using Support vector machines (Support vector classifier) with linear kernels (SVM). This method was chosen because of its ability to learn well with only a very small number of features, its robustness against the error of models, and its computational efficiency compared to other ML methods ${ }^{47}$. In addition, SVM has been shown to successfully classify cancer tissue samples based on gene expression, from microarray technology $y^{48}$ or microarray-RNAseq integrated data ${ }^{49}$.

In order to discern if the expression of these genes would be able to predict pregnancy across all the bovine breeds, the training set consisted of all the samples but the samples from a given breed, which were part of the test set. Therefore, four pairs of training-test sets were used for classification (Simmental heifers from both studies were considered together). In other words, the training sets consisted of all the samples but the ones from Holstein $(n=35)$, or Japanese Black $(n=41)$, or Charolais x Limousine $(n=40)$, or Simmental animals $(n=40)$. Then, the corresponding test set to each training set were all the samples from the Holstein $(n=17)$, or Japanese Black $(n=11)$, or Charolais $x$ Limousine $(n=12)$, or Simmental animals $(n=12)$.

The leave-one-out cross validation method was employed as the internal control for the training dataset. The implementation of the SVM with linear kernel was done with the kernlab package ${ }^{50}$, through the caret package ${ }^{51}$ for the R software ${ }^{38}$.

Exploring the biological significance of the selected biomarker genes. As a final step, we investigated the biological meaning of the 50 genes through two methods: predictions in external datasets and functional/network analysis.

Predictions in external datasets: Two datasets were selected to generate the test sets based on the endometrial expression of the 50 biomarker genes. These were GSE33030 23 and GSE16880 24 . Both studies employed the Affymetrix Bovine Genome Array (GPL2112) as platform. Only samples belonging to pregnant heifers treated or not with a progesterone device from day 3 ( $n=5$ per group), and those obtained from ovariectomized cows treated with progesterone for 6 days receiving or not an estradiol injection ( $n=3$ per group), downloaded from GSE33030 or GSE16880, respectively, were analyzed. The raw data were processed with the gcRMA package ${ }^{41}$. Data were imported into R, background corrected, and then transformed and normalized using the quantile normalization method. Next, rows of each data set were collapsed, to retain the microarray probe with the highest mean value from the group of the genes with the same Ensembl ID. The 50 genes were isolated from each dataset to be used as test sets, performing an addon batch effect adjustment of this data with the training data with the bapred package $\mathrm{e}^{52}$. The training data consisted of the batch-corrected expression of the 50 genes for all the 52 samples described in Table 1. SVM with linear kernels was used as classifier, employing the leave-one-out cross validation method as the internal control, applied with the kernlab package ${ }^{50}$, through the caret package ${ }^{51}$ for the R software ${ }^{38}$.

Functional/network analysis: A functional classification of the protein class for each gene was overview with the Panther database ${ }^{13}$. Next, in order to expand the knowledge about the genes related to the biomarker genes, a network analysis with Cytoscape V. 3.7.214 was performed.

For this, the Ensembl IDs were converted first to the corresponding human Entrez ID homologous using bioDBnet (https://biodbnet-abcc.ncifcrf.gov/db/db2db.php). Then, the GeneMania plugin ${ }^{53}$, which infers network data, was employed to generate two networks: one for the group of genes increasing in expression, and other for the genes decreasing in expression, in the $\mathrm{R}$ cows. The set of functional association data between genes was downloaded from the Homo sapiens database. The up-regulated -or down-regulated- biomarker genes were imported into the GeneMania plugin to retrieve the corresponding association network, allowing the program to find the top 100 related genes. The association data employed was genetic or physical interaction (i.e., two genes are functionally associated, if the effects of perturbing one gene were found to be modified by perturbations to a second gene, or if their products were found to interact in a protein-protein interaction study) or if the genes were in the same reaction within a pathway. Finally, the BinGO plugin ${ }^{54}$ was applied to find the statistically overrepresented biological processes in the resulting networks. 


\section{Data availability}

All data are fully resourced from public NCBI GEO databases.

Received: 17 April 2020; Accepted: 7 September 2020

Published online: 12 October 2020

\section{References}

1. Suravajhala, P., Kogelman, L. J. \& Kadarmideen, H. N. Multi-omic data integration and analysis using systems genomics approaches: methods and applications in animal production, health and welfare. Genet Sel. Evol. 48, 38 (2016).

2. Kadarmideen, H. N. \& Mazzoni, G. Transcriptomics-genomics data integration and expression quantitative trait loci analyses in oocyte donors and embryo recipients for improving invitro production of dairy cattle embryos. Reprod. Fertil. Dev. 31, 55-67 (2018).

3. Ghaffari, M. H. et al. Metabolomics meets machine learning: Longitudinal metabolite profiling in serum of normal versus overconditioned cows and pathway analysis. J. Dairy Sci. 102, 11561-11585 (2019).

4. Libbrecht, M. W. \& Noble, W. S. Machine learning applications in genetics and genomics. Nat. Rev. Genet. 16, 321-332 (2015).

5. Lagani, V., Karozou, A. D., Gomez-Cabrero, D., Silberberg, G. \& Tsamardinos, I. A comparative evaluation of data-merging and meta-analysis methods for reconstructing gene-gene interactions. BMC Bioinform. 17(Suppl 5), 194 (2016).

6. Spencer, T. E. \& Bazer, F. W. Uterine and placental factors regulating conceptus growth in domestic animals. J. Anim. Sci. 82 E-Suppl, E4-E13 (2004).

7. Mazzoni, G. et al. Characterization of the endometrial transcriptome in early diestrus influencing pregnancy status in dairy cattle after transfer of in vitro-produced embryos. Physiol. Genomics 52, 269-279 (2020).

8. Binelli, M. et al. The transcriptome signature of the receptive bovine uterus determined at early gestation. PLoS ONE 10, e0122874 (2015).

9. Ponsuksili, S. et al. Gene expression and DNA-methylation of bovine pretransfer endometrium depending on its receptivity after in vitro-produced embryo transfer. PLoS ONE 7, e42402 (2012).

10. Salilew-Wondim, D. et al. Aberrant placenta gene expression pattern in bovine pregnancies established after transfer of cloned or in vitro produced embryos. Physiol. Genomics 45, 28-46 (2013).

11. Killeen, A. P. et al. Global gene expression in endometrium of high and low fertility heifers during the mid-luteal phase of the estrous cycle. BMC Genomics 15, 234 (2014).

12. Leclercq, M. et al. Large-scale automatic feature selection for biomarker discovery in high-dimensional OMICs data. Front. Genet 10, 452 (2019).

13. Thomas, P. D. et al. PANTHER: a library of protein families and subfamilies indexed by function. Genome Res. 13, 2129-2141 (2003).

14. Shannon, P. et al. Cytoscape: a software environment for integrated models of biomolecular interaction networks. Genome Res. 13, 2498-2504 (2003).

15. Moran, B., Butler, S. T., Moore, S. G., MacHugh, D. E. \& Creevey, C. J. Differential gene expression in the endometrium reveals cytoskeletal and immunological genes in lactating dairy cows genetically divergent for fertility traits. Reprod. Fertil. Dev. 29, 274-282 (2017).

16. Clough, E. \& Barrett, T. The gene expression omnibus database. Methods Mol. Biol. 1418, 93-110 (2016).

17. Rabaglino, M. B. \& Conrad, K. P. Evidence for shared molecular pathways of dysregulated decidualization in preeclampsia and endometrial disorders revealed by microarray data integration. FASEB J. 33, 11682-11695 (2019).

18. Diskin, M. G. \& Morris, D. G. Embryonic and early foetal losses in cattle and other ruminants. Reprod. Domest Anim. 43(Suppl 2), 260-267 (2008).

19. Kiser, J. N. et al. Validation of 46 loci associated with female fertility traits in cattle. BMC Genomics 20, 576 (2019).

20. Neupane, M. et al. Loci and pathways associated with uterine capacity for pregnancy and fertility in beef cattle. PLoS ONE 12, e0188997 (2017).

21. Spencer, T. E., Forde, N. \& Lonergan, P. Insights into conceptus elongation and establishment of pregnancy in ruminants. Reprod. Fertil. Dev. 29, 84-100 (2016).

22. Spencer, T. E., Forde, N. \& Lonergan, P. The role of progesterone and conceptus-derived factors in uterine biology during early pregnancy in ruminants. J. Dairy Sci. 99, 5941-5950 (2016).

23. Forde, N. et al. Progesterone-regulated changes in endometrial gene expression contribute to advanced conceptus development in cattle. Biol. Reprod. 81, 784-794 (2009).

24. Shimizu, T. et al. Actions and interactions of progesterone and estrogen on transcriptome profiles of the bovine endometrium. Physiol. Genomics 42A, 290-300 (2010).

25. Smith, J. F., Fairclough, R. J., Payne, E. \& Peterson, A. J. Plasma hormone levels in the cow: I. Changes in progesterone and oestrogen during the normal oestrous cycle. N. Z. J. Agric. Res. 18, 123-129 (1975).

26. Chen, J. The cell-cycle arrest and apoptotic functions of p53 in tumor initiation and progression. Cold Spring Harb. Perspect. Med. 6, a026104 (2016).

27. Mercer, W. E. Checking on the cell cycle. J. Cell Biochem. Suppl. 30-31, 50-54 (1998).

28. Kang, H. J. \& Rosenwaks, Z. p53 and reproduction. Fertil. Steril. 109, 39-43 (2018).

29. Zamparini, A. L. et al. Hex acts with beta-catenin to regulate anteroposterior patterning via a Groucho-related co-repressor and Nodal. Development 133, 3709-3722 (2006).

30. Bainbridge, T. W. et al. Evolutionary divergence in the catalytic activity of the CAM-1, ROR1 and ROR2 kinase domains. PLoS ONE 9, e102695 (2014).

31. Kim, T. W. et al. Ctbp2-mediated $\beta$-catenin regulation is required for exit from pluripotency. Exp. Mol. Med. 49, e385 (2017).

32. Tribulo, P., Leão, B. C. D. S., Lehloenya, K. C., Mingoti, G. Z. \& Hansen, P. J. Consequences of endogenous and exogenous WNT signaling for development of the preimplantation bovine embryo. Biol. Reprod. 96, 1129-1141 (2017).

33. Honma, S. et al. Dec1 and Dec2 are regulators of the mammalian molecular clock. Nature 419, 841-844 (2002).

34. Nakashima, A. et al. DEC1 modulates the circadian phase of clock gene expression. Mol. Cell Biol. 28, 4080-4092 (2008).

35. Sen, A. \& Hoffmann, H. M. Role of core circadian clock genes in hormone release and target tissue sensitivity in the reproductive axis. Mol. Cell Endocrinol. 501, 110655 (2020).

36. Barrett, T. et al. NCBI GEO: archive for functional genomics data sets-update. Nucl. Acids Res. 41, D991-D995 (2013).

37. Edgar, R., Domrachev, M. \& Lash, A. E. Gene Expression Omnibus: NCBI gene expression and hybridization array data repository. Nucl. Acids Res. 30, 207-210 (2002).

38. R Core Team. R: A Language and Environment for Statistical Computing. Vienna, Austria. (2020). https://www.R-project.org/.

39. Huber, W., von Heydebreck, A., Sueltmann, H., Poustka, A. \& Vingron, M. Parameter estimation for the calibration and variance stabilization of microarray data. Stat. Appl. Genet. Mol. Biol.2, Article3 (2003).

40. Love, M. I., Huber, W. \& Anders, S. Moderated estimation of fold change and dispersion for RNA-seq data with DESeq2. Genome Biol. 15, 550 (2014). 
41. Wu, J., Irizarry, R. \& Gentry, W. C. F. J. M. J. gcrma: Background Adjustment Using Sequence Information. (2017).

42. Ritchie, M. E. et al. limma powers differential expression analyses for RNA-sequencing and microarray studies. Nucl. Acids Res 43, e47 (2015).

43. Johnson, W. E., Li, C. \& Rabinovic, A. Adjusting batch effects in microarray expression data using empirical Bayes methods. Biostatistics 8, 118-127 (2007).

44. Su, S. et al. Glimma: interactive graphics for gene expression analysis. Bioinformatics 33, 2050-2052 (2017).

45. de Hoon, M. J., Imoto, S., Nolan, J. \& Miyano, S. Open source clustering software. Bioinformatics 20, 1453-1454 (2004).

46. Saldanha, A. J. Java Treeview-extensible visualization of microarray data. Bioinformatics 20, 3246-3248 (2004).

47. Kecman, V. Support Vector Machines: An introduction. In Support Vector Machines: Theory and Applications. Studies in Fuzziness and Soft Computing (ed. Wang, L.) 1-47 (Springer, 2005).

48. Furey, T. S. et al. Support vector machine classification and validation of cancer tissue samples using microarray expression data. Bioinformatics 16, 906-914 (2000).

49. Huang, C. et al. Machine learning predicts individual cancer patient responses to therapeutic drugs with high accuracy. Sci. Rep. 8, 16444 (2018).

50. Karatzoglou, A., Smola, A., Hornik, K. \& Zeileis, A. kernlab-An S4 Package for Kernel Methods in R. J. Stat. Softw. 11, 1-20 (2004).

51. Kuhn, M. Building predictive models in R using the caret package. J. Stat. Softw. 28, 1-26 (2008).

52. Hornung, R., Causeur, D., Bernau, C. \& Boulesteix, A. L. Improving cross-study prediction through addon batch effect adjustment or addon normalization. Bioinformatics 33, 397-404 (2017).

53. Montojo, J. et al. GeneMANIA Cytoscape plugin: fast gene function predictions on the desktop. Bioinformatics 26, 2927-2928 (2010).

54. Maere, S., Heymans, K. \& Kuiper, M. BiNGO: a Cytoscape plugin to assess overrepresentation of gene ontology categories in biological networks. Bioinformatics 21, 3448-3449 (2005).

55. Salilew-Wondim, D. et al. Bovine pretransfer endometrium and embryo transcriptome fingerprints as predictors of pregnancy success after embryo transfer. Physiol. Genomics 42, 201-218 (2010).

\section{Acknowledgements}

The MBR's appointment at the Technical University of Denmark was funded by the grant from Innovation Fund Denmark (7045-00013B).

\section{Author contributions}

M.B.R. developed the pipeline for this study, integrated and analyzed transcriptomic datasets using ML methods and interpreted the results. M.B.R. wrote first draft. H.N.K. conceived the application of ML methods and improved the draft of this manuscript. Both authors read and approved the final version.

\section{Competing interests}

The authors declare no competing interests.

\section{Additional information}

Supplementary information is available for this paper at https://doi.org/10.1038/s41598-020-72988-3.

Correspondence and requests for materials should be addressed to H.N.K.

Reprints and permissions information is available at www.nature.com/reprints.

Publisher's note Springer Nature remains neutral with regard to jurisdictional claims in published maps and institutional affiliations.

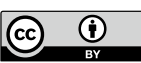

Open Access This article is licensed under a Creative Commons Attribution 4.0 International License, which permits use, sharing, adaptation, distribution and reproduction in any medium or format, as long as you give appropriate credit to the original author(s) and the source, provide a link to the Creative Commons licence, and indicate if changes were made. The images or other third party material in this article are included in the article's Creative Commons licence, unless indicated otherwise in a credit line to the material. If material is not included in the article's Creative Commons licence and your intended use is not permitted by statutory regulation or exceeds the permitted use, you will need to obtain permission directly from the copyright holder. To view a copy of this licence, visit http://creativecommons.org/licenses/by/4.0/.

(C) The Author(s) 2020 